Perspectiva Geográfica

ISSN 0123-3769 (Impreso) - 2500-8684 (En línea)

Vol. 21 No. 1 de 2016

Enero - Junio

pp. 63-90

\title{
Análisis de la distribución espacial y temporal de la precipitación en la cuenca del río Chicú, Sabana de Bogotá, Colombia ${ }^{1}$
}

\author{
Spatial and Temporal Precipitation Distribution Analysis at \\ Chicú River Basin, Bogotás High Altitude Savannah, Colombia
}

Cristian Camilo López Velandia ${ }^{2}$

Para citar este artículo utilice el nombre completo así:

López, C. (2016). Análisis de la distribución espacial y temporal de la precipitación en la cuenca del río Chicú, Sabana de Bogotá, Colombia. Perspectiva Geográfica, 21(1), 63-90.

\section{Resumen}

Se analizó la variabilidad local y espacio-temporal de la precipitación en la cuenca del río Chicú, como insumo en la detección de posibles impactos del cambio climático y el diseño de medidas de adaptación y mitigación a nivel municipal; para ello se obtuvo la información histórica de precipitación, recopilada de las estaciones hidrometeorológicas de la región, y se realizó el análisis de calidad y estimación de datos faltantes, con el propósito de generar mapas de distribución espacial de la precipitación para la cuenca. Se presenta una precipitación media anual de 853 mm, para la estación climatológica Gja. Providencia (1987-2012), influenciada por la Zona de Interconfluencia Tropical; la distribución de la precipitación media mensual es de carácter bimodal: dos periodos lluviosos, en los meses de abril-mayo $(101,9 \pm 15,6 \mathrm{~mm})$ y octubre-

\footnotetext{
1 Este artículo es resultado de la Tesis de Maestría sin publicar titulada Evaluación ambiental y química del recurso hídrico subterráneo de la Cuenca del Río Chicú, Bogotá, Colombia.

2 Licenciado en Química, Universidad Pedagógica de Colombia. Candidato Msc. en Ecohidrología, Universidad Nacional de la Plata. cristianlopezlic@gmail.com
} 
noviembre $(98,9 \pm 19,5 \mathrm{~mm})$, produciendo variaciones significativas en torno a la distribución espacial de la precipitación en la zona de estudio.

Palabras clave: cuenca del río Chicú, distribución espacial de lluvias, precipitación temporal de lluvias y variabilidad espacial.

\begin{abstract}
In this paper the spatio-temporal variability of rainfall in the Chicú river basin was analyzed locally, in order to use it as input to identify potential impacts of climate change and mitigation design and adaptation measures at the municipal or rural level. Historical precipitation information was collected by hydro-meteorological stations present in the region, performing quality analysis and estimation of missing data, in order to generate maps of spatial distribution of rainfall for the region. This region presents an average annual rainfall of 853 $\mathrm{mm}$ for the "Providencia" rural weather station (1987-2012). It is influenced by the Inter-confluence of the Tropical Zone. The average monthly precipitation is of bimodal character, generating two rainy periods, the first in the months of April-May $(101.9 \pm 15.6 \mathrm{~mm})$ and the second in October-November $(98.9 \pm 19.5$ $\mathrm{mm}$ ), producing significant variations around the spatial distribution of rainfall in the analyzed area during the months studied.
\end{abstract}

Keywords: Chicú river basing, rainfall spatial and temporal variability, precipitation. 


\section{Introducción}

El conocimiento de la distribución espacial y del ciclo anual de la precipitación es de vital importancia para un país, desde el punto de vista social y económico (Mejía et al., 1999); por ejemplo, en las llanuras suramericanas, cualquier variación en torno a los regímenes de precipitación y temperatura provoca significativas consecuencias sobre su producción (Nogués-Paegle y Mo, 1997) y, por lo tanto, sobre la alimentación en el continente.

Colombia, localizada en el extremo noroeste de América del Sur, aproximadamente entre $4^{\circ}$ latitud Sur y $13^{\circ}$ latitud Norte, es un país tropical intramontañoso que presenta gran variedad de climas y amplia variación y contraste entre los valores de los elementos climatológicos (Eslava, 1993). Esta variabilidad está determinada, principalmente, por la influencia de los vientos alisios y de la oscilación de la Zona de Convergencia Intertropical (ZICT), que condiciona las propiedades físicas de la atmósfera; por la influencia de los océanos Pacífico y Atlántico, que proveen humedad; por la conformación fisiográfica, que incluye cadenas montañosas (cordillera de los Andes), con la presencia de valles intramontanos y vertientes de cordillera que presentan marcadas diferencias climáticas, y, además, por el efecto de la circulación propia de la cuenca del Amazonas y de la vegetación y su fuerte influencia sobre los procesos de escurrimiento superficial, humedad de suelo y evapotranspiración (Eslava, 1993; Mejía et al. 1999).

Dada la ubicación geográfica de Colombia, la mayoría de su territorio se encuentra influenciado por la ZICT, zona de la atmósfera en la que confluyen dos masas de aire con baja presión relativa, situada, aproximadamente, paralela al Ecuador y ubicada entre dos núcleos de alta presión atmosférica; la diferencia de presión entre los núcleos y la ZICT provocan movimientos horizontales de esta, induciendo a que las áreas que no están bajo la influencia de la ZITC presenten, en términos generales, un clima seco y soleado; por su parte, bajo la influencia de la ZITC se presenta cielo nuboso, con la aparición de abundantes lluvias (Eslava, 1993; Nogués-Paegle \& Mo, 1997). Este efecto de migración meridional de la ZITC en términos temporales, y su interacción con las circulaciones de los océanos 
Pacífico y Atlántico y de la cuenca del Amazonas y el Orinoco es uno de los mecanismos utilizados para explicar la variabilidad anual o semianual de la precipitación y su distribución espacial bimodal o unimodal (Glandz, 2000; Waylen, Quesada \& Caviedes, 1996; Poveda, Waylen \& Pulwarty, 2006; García, Piñeros, Bernal y Ardilla, 2012).

Tradicionalmente, se ha dado por supuesto que la distribución espacial de la lluvia sobre pequeñas áreas es uniforme, utilizándose el registro de un solo pluviómetro para caracterizar las precipitaciones de un determinado espacio (Patrick y Stephenson, 1990); no obstante, la extrapolación del registro de un solo pluviómetro al conjunto de toda una cuenca puede, en ocasiones, no ser representativo del conjunto y, por lo tanto, alterar el resultado final (Barbancho, 1998). Por ello, el objetivo del presente trabajo es cuantificar y analizar la variabilidad espacial de la cuenca en estudio, que pueda ser utilizada para la interpolación entre observaciones y el mapeo de la distribución espacial de la precipitación, con el fin de comprender la variabilidad climática en torno a los regímenes de precipitación en la región en estudio.

\section{Planteamiento del problema}

Dada la amplia variabilidad climática de Colombia y las respuestas hidrológicas complejas que se desarrollan en la región, sumado a los efectos del calentamiento global, vinculado con el cambio climático, surge la necesidad imperante de información hidrometeorológica a escala local para la toma de decisiones (García, Piñeros, Bernal y Ardilla, 2012) en torno al comportamiento de los recursos hídricos superficiales y subterráneos, ante la presión antrópica, por la demanda de las actividades agropecuarias y domésticas que se desarrollan en la región respecto al uso de agua, los efectos del cambio climático y la variabilidad climática, los cuales generan efectos sobre su disponibilidad para satisfacer las necesidades de la población.

Colombia no ha sido ajena a los cambios dramáticos del clima global y a los efectos del calentamiento global, entendido como el aumento progresivo de la temperatura media del aire, que puede alterar el patrón climático global (Environmental Protection Agency, 2010). En el año 2010, el país vivió uno de los periodos más críticos en términos de anomalías en precipitación frente al 
promedio anual; el mes de julio fue el más lluvioso de los últimos 30 años, debido a que persistieron las lluvias por encima de lo normal en la región Caribe, Andina y Pacífica, $y$ en diciembre se presentaron inundaciones severas por los efectos de la Niña (García, Piñeros, Bernal y Ardila, 2012). Por otra parte, a nivel departamental (Cundinamarca) se espera que los efectos del calentamiento global generen aumentos de precipitación, con valores de entre $10 \%$ y $30 \%$ adicionales a los valores actuales, cuyos principales efectos podrán presentarse en el sector agrícola, debido los cambios acentuados de temperatura, así como en la persistencia de plagas asociadas al aumento de precipitación para las zonas en las cuales este valor se eleva (Ideam, PNUD, MADS, DNP, Cancillería, 2015). Dicha alteración en el ciclo hidrológico podría representar mayores impactos en los humanos $\mathrm{y}$ en los sistemas ambientales que los mismos cambios en la temperatura (Mcelwain \& Sweeney, 2003), dado el aumento en la frecuencia de eventos extremos de precipitación y sequía en diferentes regiones del mundo.

Pese a la gran disponibilidad de datos a nivel mundial y a la publi- cación de los modelos climáticos globales, que representan un insumo importante para los decisores de políticas a escala mundial o regional, a nivel de mesoclima, esta información es insuficiente para la toma de decisiones a nivel municipal o de finca (Rojas, Arce, Peña, Boshell y Ayarza, 2010). Por lo tanto, este trabajo pretende proveer información a escala local en torno a la variabilidad espacial y temporal de la precipitación en la cuenca del río Chicú, ubicada entre los municipios de Tabio y Tenjo, en el departamento de Cundinamarca, Colombia, para que pueda ser utilizada como insumo en la detección de posibles impactos del cambio climático y para el diseño de medidas de adaptación y mitigación en la región.

\section{3. Área de estudio}

La región en estudio, denominada cuenca del río Chicú (Figura 1), conformada por los municipios de Tabio y Tenjo, se encuentra dentro de la Sabana de Bogotá, Colombia; es un sinclinorio fallado con rumbo SSW-NNE en el centro y ubicada geográficamente en la cresta de la cordillera Oriental (Montoya y Reyes, 2005; Lobo-guerrero, 1992); se encuentra limitada por 


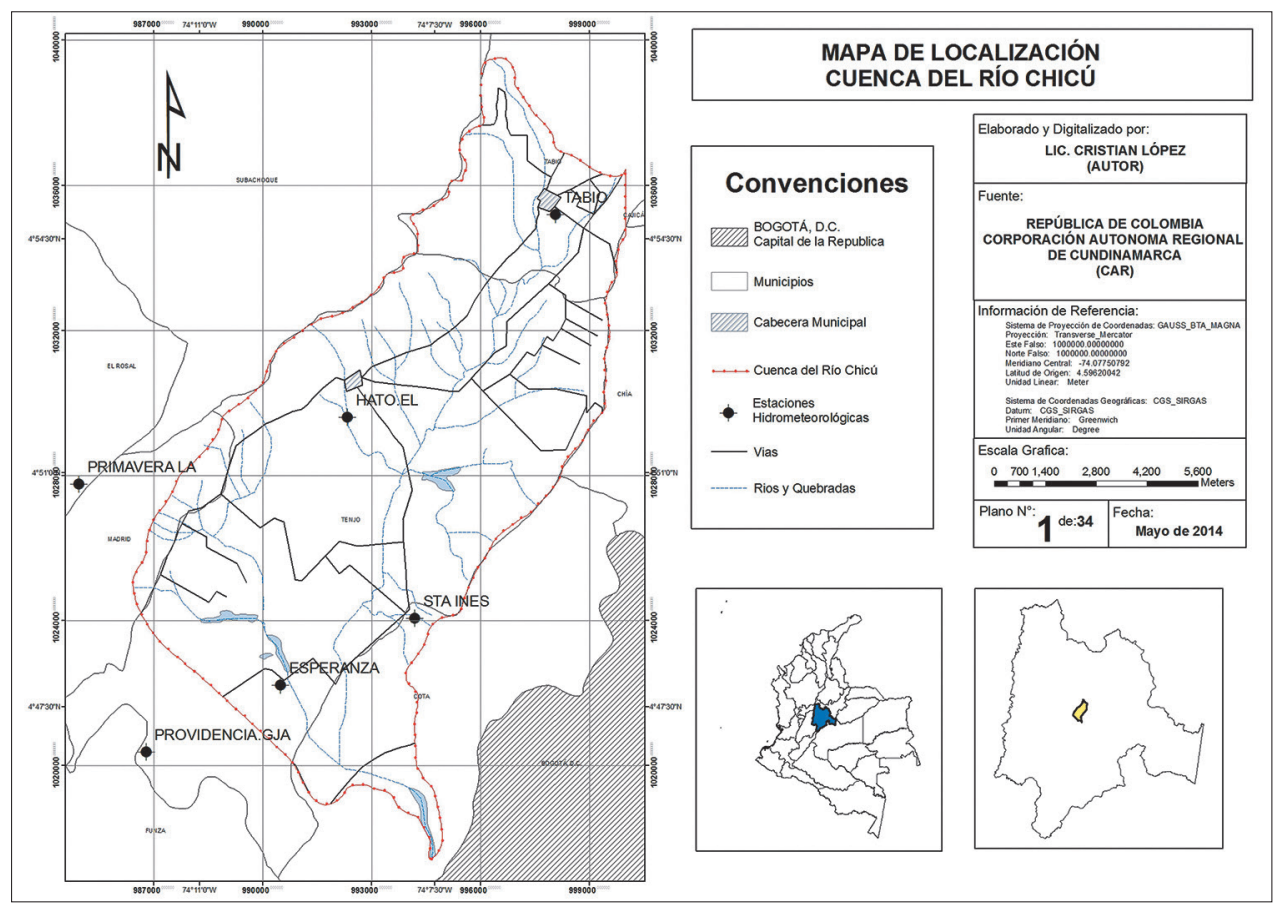

Figura 1. Mapa de localización: cuenca del río Chicú

Fuente: Elaboración propia.

la serranía de Cota, al este, y el Sabana y Chía, que conforman la anticlinal de Tabio-Tenjo, al oeste, zona plana (Robles y Sáenz, 1990; y delimitada por tres unidades Lobo-guerrero, 1992; Robles, fisiográficas: una zona plana, que Martínez, Hincapié y Álvarez, conforma un valle intramontano, 1991), y, por último, una zona de extenso y plano, localizada en pendientes suaves, ubicada en la el centro de la cuenca; una zona zona de piedemonte. Los puntos montañosa, que bordea el valle, extremos de la zona de estudio que se inicia al este del altiplano se ubican con las siguientes y se prolonga hacia el sur hasta coordenadas geográficas (Tabla 1). introducirse bajo los sedimentos 
Tabla 1. Límites de la zona de estudio

\begin{tabular}{|c|c|c|}
\hline Límite & Coordenadas & Descripción \\
\hline Norte & $\begin{array}{l}4^{\circ} 57^{\prime} 12.78^{\prime} \mathrm{N} \\
74^{\circ} 06^{\prime} 33.66^{\prime} \mathrm{W}\end{array}$ & $\begin{array}{l}\text { Ubicado sobre la serranía de Tabio-Tenjo, en el } \\
\text { municipio de Tabio, limitando con el municipio de } \\
\text { Subachoque. }\end{array}$ \\
\hline Sur & $\begin{array}{l}4^{\circ} 45^{\prime} 14.533^{\prime} \mathrm{N} \\
74^{\circ} 07^{\prime} 31.92^{\prime \prime} \mathrm{W}\end{array}$ & $\begin{array}{l}\text { Zona de desagüe de la cuenca del río Chicú sobre el } \\
\text { río Bogotá. }\end{array}$ \\
\hline Este & $\begin{array}{l}4^{\circ} 51^{\prime} 57.25^{\prime \prime} \mathrm{N} \\
74^{\circ} 04^{\prime} 57.64^{\prime \prime} \mathrm{W}\end{array}$ & $\begin{array}{l}\text { Ubicado sobre la serranía de Cota, en el municipio de } \\
\text { Tenjo, limitando con el municipio de Cota. }\end{array}$ \\
\hline Oeste & $\begin{array}{l}4^{\circ} 50^{\prime} 15.28^{\prime \prime} \mathrm{N} \\
74^{\circ} 11^{\prime} 42.61^{\prime \prime} \mathrm{W}\end{array}$ & $\begin{array}{l}\text { Ubicado sobre la serranía de Tabio-Tenjo, en el } \\
\text { municipio de Tenjo, limitando con el municipio de El } \\
\text { Rosal. }\end{array}$ \\
\hline
\end{tabular}

Fuente: Elaboración propia.

El área de estudio se encuentra entre los 2.510 msnm y los $3.140 \mathrm{msnm}$, con un área total de 147,93 kilómetros cuadrados.

Tabla 2. Estaciones climatológicas e hidrometeorológicas en la zona de estudio

\begin{tabular}{|c|c|c|c|c|c|c|c|c|}
\hline \multirow{4}{*}{ Nombre } & \multirow{4}{*}{ Tipo } & \multicolumn{5}{|c|}{ LOCALIZACIÓN } & \multirow{4}{*}{$\begin{array}{l}\text { Periodo de } \\
\text { registro }\end{array}$} & \multirow{4}{*}{ Admo. } \\
\hline & & \multicolumn{2}{|c|}{$\begin{array}{c}\text { Proyección } \\
\text { TM-Sistema } \\
\text { de Referencia } \\
\text { MARGA - SIRGAS }\end{array}$} & \multicolumn{2}{|c|}{$\begin{array}{c}\text { Proyección Elipsoidal } \\
\text { - Sistema de Referencia } \\
\text { MARGA - SIRGAS }\end{array}$} & \multirow[t]{2}{*}{ Elev. } & & \\
\hline & & $X$ & $\mathrm{Y}$ & Lat. & Long. & & & \\
\hline & & Este & Norte & Norte & Oeste & m.s.n.m. & & \\
\hline Sta. Inés & $\mathrm{PM}$ & 993400 & 1023000 & $74^{\circ} 08^{\prime} 12,2^{\prime \prime}$ & $4^{\circ} 48^{\prime} 15,1^{\prime \prime}$ & 2550 & $1980-2013$ & CAR \\
\hline Tabio & $\mathrm{CP}$ & 998070 & 1035180 & $74^{\circ} 05^{\prime} 41,7^{\prime \prime}$ & $4^{\circ} 54^{\prime} 51,6^{\prime \prime}$ & 2620 & $1970-2013$ & CAR \\
\hline La Primavera & $\mathrm{CP}$ & 985090 & 1029900 & $74^{\circ} 12^{\prime} 42,9^{\prime \prime}$ & $4^{\circ} 51^{\prime} 59,7^{\prime \prime}$ & 2590 & $1965-2013$ & CAR \\
\hline La Ramada & $\mathrm{CP}$ & 989110 & 1011430 & $74^{\circ} 10^{\prime} 32,4^{\prime \prime}$ & $4^{\circ} 41^{\prime} 58,4$ '” & 2545 & $1938-2013$ & CAR \\
\hline El Hato & PM & 991531 & 1029909 & $74^{\circ} 09^{\prime} 13,9^{\prime \prime}$ & $4^{\circ} 52^{\prime} 0,0 ’$ & 2575 & $1978-2013$ & IDEAM \\
\hline Esperanza & PM & 988634 & 1022777 & $74^{\circ} 10^{\prime} 47,9^{\prime \prime}$ & $4^{\circ} 48^{\prime} 7.8^{\prime \prime}$ & 2555 & $1974-2013$ & IDEAM \\
\hline $\begin{array}{l}\text { Gja. } \\
\text { Providencia }\end{array}$ & PM & 986310 & 1021696 & $74^{\circ} 12^{\prime} 3,3{ }^{\prime \prime}$ & $4^{\circ} 47^{\prime} 32,6^{\prime \prime}$ & 2560 & $1983-2013$ & IDEAM \\
\hline
\end{tabular}

PM: Pluviométrica; CP: Climatológica Principal; Lat.: Latitud; Long.: Longitud; Elev.: Elevación; m.s.n.m.: Metros sobre el nivel del mar; Admo.: Administrado.

Fuente: Elaboración propia. 
Con el fin de realizar una breve descripción del comportamiento espacial y temporal de la precipitación en la región de estudio, se obtuvo la información histórica de precipitación recopilada de las estaciones controladas por la CAR y el IDEAM (Tabla 2), a la cual se le realizó el respectivo análisis de calidad y la estimación de datos faltantes en las series pluviométricas, a través de la metodología de regresión lineal múltiple y del método del cociente normal. Con las series completas, y teniendo en cuenta los promedios intermensuales (1987-2012; 26 años) de cada estación utilizada, se generaron los mapas de distribución espacial de la precipitación para la región en estudio, utilizando el software Surfer ${ }^{\circledR} 10$. Se generaron las isohietas de precipitación media intermensual y de los meses de julio y octubre, debido a que en estos meses, por efecto de la ZICT, se presentan en la zona épocas de déficit y exceso hídrico, respectivamente.

Para la generación de los mapas se realizó la determinación y ajuste de los parámetros para la resolución de la metodología de curvatura mínima (Minimum Curvature) para cada uno de los datos de interés.

\section{Metodología}

\subsection{Métodos de estimación de da- tos faltantes}

Se utilizaron dos métodos de estimación de datos faltantes, de acuerdo con las características de correlación entre las estaciones que presentan series de datos de comportamiento similar dentro de la misma área de influencia topoclimática (referencia) y la estación cuya serie tiene datos faltantes (estudio). Para las estaciones con coeficientes de Pearson mayores a \pm 0.80 se utilizó el método de Regresión Lineal Múltiple, y para las estaciones que presentaron coeficientes menores a $\pm 0,80$ se utilizó el método del Cociente normal.

\section{Método de Regresión Lineal Múl-} tiple: El modelo de regresión lineal múltiple es idéntico al modelo de regresión lineal simple, con la única diferencia de que aparecen más variables explicativas (ecuación 1):

$$
y=\beta_{0}+\beta_{1} X_{1}+\beta_{2} X_{2}+\cdots+\beta_{k} X_{k}
$$

Para aplicar este método se normalizaron las series de datos de las estaciones presentes en la zona, utilizando su raíz cuadrada y eliminando los datos ausentes de cada 
una de las series utilizadas; se utilizó el software R.

Como consideraciones para el modelo determinado, si los coeficientes de regresión son diferentes de cero estadísticamente, según prueba de $t$ al $5 \%$, con un coeficiente de determinación mayor del $75 \%$, y se cumplen los siguientes supuestos: linealidad del modelo, varianza constante, independencia y normalidad de los errores, se tendría un método estadístico para estimar los datos faltantes de la serie de estudio, simplemente reemplazando en la expresión obtenida, una vez identificado el tiempo en el cual falta el dato en la serie de tiempo, el valor correspondiente al mismo tiempo de ocurrencia del dato faltante de la serie de referencia (Medina, 2008).
Método de Cociente Normal: Para las restantes estaciones, cuyo coeficiente de correlación no supera la barrera del valor $\pm 0,8$, se utilizó este método, que se aplica cuando la precipitación anual de cualquiera de las estaciones de referencia difiere más del $10 \%$ de la estación con el dato faltante (Medina, 2008). Para ello, se tomó la precipitación anual de cada una de las tres estaciones con mayor correlación y se multiplicó por la relación (cociente) entre la precipitación anual de la estación con el dato faltante y la precipitación anual de la estación de referencia (corrección estimada); luego, se obtuvo el valor faltante como la suma del producto de precipitación ocurrida en la estación de referencia y corrección estimada, dividida por 3 , es decir (ecuación 2):

$$
D_{j}=\frac{a_{j} p_{A}+b_{j} p_{B}+c_{j} p_{C}}{3}
$$

Donde:

$$
\mathrm{p}_{\mathrm{x}}=\frac{\text { Precipitación media mensual estación dato faltante }}{\text { Precipitación media mensual estación de referencia } X}
$$

$\mathrm{Dj}=$ Precipitación estimada para el mes $\mathrm{j}$

aj, bj, cj = Precipitación registrada en las tres estaciones de referencia el mes j 


\subsection{Método de interpolación espacial de curvatura mínima}

Con el fin de realizar los mapas de distribución espacial de la precipitación, se utilizó la metodología de la curvatura mínima, que es ampliamente utilizada en las ciencias de la Tierra; con esta metodología, la superficie interpolada generada es análoga a una placa delgada linealmente elástica que pasa a través de cada uno de los valores de los datos, con una cantidad mínima de flexión, generando una superficie lisa al tratar de honrar sus datos lo más fielmente posible; no obstante, esta no es un interpolador exacto. El método de curvatura mínima requiere de al menos 4 puntos de datos (Golden Software, Inc., 2002; Yang, Kao, Lee y Hung, 2004).

Este método produce una superficie, aplicando repetidamente una ecuación sobre la grilla en un intento de suavizarla. Cada paso sobre la grilla es contado como una iteración. El valor del nodo en la grilla es recalculado hasta que es menor que la tolerancia específica (residuo máximo), o cuando el número máximo de iteraciones es alcanzado (Briggs, 1974; Smith y Wessel, 1990; Golden Software,
Inc., 2002), siendo esta rutina ajustada en primera medida a un modelo plano simple usando la regresión de mínimos cuadrados (ecuación 3) (Smith \& Wessel, 1990):

$$
A X+B Y+C=Z(X, Y)
$$

Con el fin de generar una grilla final usando el método de curvatura mínima, se realizaron cuatro pasos (Golden Software, Inc., 2002):

1. El modelo de regresión de mínimos cuadrados es ajustado a los datos.

2. Los valores del modelo de regresión plana en las localizaciones de los datos son sustraídos desde los valores de los datos; esto deja un grupo de valores residuales.

3. El algoritmo de la curvatura mínima es usado para interpolar los residuos en los nodos de la grilla.

4. Los valores del modelo de regresión plana en los nodos de la grilla son adicionados a los residuos interpolados, dejando una superficie interpolada final.

Los nodos fijos son definidos como el promedio de los valores vecinos observados, considerando un rectángulo del tamaño y forma de la celda de la grilla. El vecindario de 
los nodos es definido por la grilla de nodos centrados en el rectángulo. Si existe algún dato observado dentro del vecindario del nodo, el valor de este es fijado como el promedio aritmético del valor contenido. Esta metodología genera la superficie que interpola los datos disponibles y soluciona la ecuación diferencial biharmónica modificada con tensión (ecuación 4):

$$
\left(1-T_{i}\right) \nabla^{2}\left(\nabla^{2} Z\right)-\left(T_{i}\right) \nabla^{2} Z=0
$$

Se encuentran tres ecuaciones asociadas a las condiciones de borde:

En los bordes (ecuación 5)

$$
\left(1-T_{b}\right) \frac{\delta^{2} Z}{\delta n^{2}}+\left(T_{b}\right) \frac{\delta Z}{\delta n}=0
$$

En los bordes (ecuación 6)

$$
\frac{\delta\left(\nabla^{2} Z\right)}{\delta n}=0
$$

En las esquinas (ecuación 7)

$$
\frac{\delta^{2} Z}{\delta x \delta y}=0
$$

\section{Donde}

$\begin{array}{cl}\nabla^{2} & \text { es el operador laplaciano } \\ n & \text { es el borde normal } \\ T_{i} & \text { es la tensión interna } \\ T_{b} & \text { es la tensión de borde }\end{array}$

En el programa Surfer ${ }^{\circledR} 10$ es posible controlar el criterio de convergencia para la metodología de curvatura mínima de la siguiente manera (Golden Software, Inc., 2002):

- El parámetro de residuo máximo, que tiene las mismas unidades que los datos. Un valor apropiado es, aproximadamente, el $10 \%$ de la precisión del dato. Las iteraciones continuarán hasta que la corrección del nodo para la iteración dada sea menor que el valor de residuo máximo.

- El parámetro de máxima iteración, que debe estar ajustado a una o dos veces el número de nodos de grilla generados en el archivo de grilla. Por ejemplo, cuando se generan grillas de 50 por 50, usando la curvatura mínima, el valor de iteración máxima debe estar ajustado entre $2.500 \mathrm{y}$ 5.000 .

- La tensión interna, usada para controlar la cantidad de esta inclinación en el interior: a mayor tensión, mejor la inclinación. La tensión de borde controla la cantidad de inclinación en los bordes. Por defecto, en Surfer ${ }^{\circledR} 10$ la tensión interna y la tensión de borde son ajustadas a cero. 
- El factor de relajación, cuyo valor genérico es 1.0; a mayor valor (cercano a dos) permite una convergencia rápida del algoritmo de curvatura mínima, pero es más seguro que este no converja del todo, y a menor valor (cercano a cero) es más seguro que el algoritmo de mínima curvatura converja, pero el algoritmo es lento. El factor óptimo de relajación es derivado a través del ensayo-error (Press, Flannery, Teukolsky y Vetterling, 1988).

- La Anisotropía, que es un fenómeno natural creado por procesos físicos que, frecuentemente, tienen orientaciones preferenciales; este parámetro permite captar las tendencias en los datos y las toma en cuenta durante los procesos de interpolación espacial. Los ajustes de la anisotropía para el método de curvatura mínima incluye el radio de anisotropía, siendo este el máximo rango dividido por el rango mínimo.

Una relación de anisotropía menor que dos es considerada moderada, mientras que una mayor que cuatro es considerada severa. Típicamente, cuando la relación de anisotropía es mayor que tres, su efecto es fácilmente visible en los mapas basados en grillas.

\section{Resultados y discusión}

La región de estudio presenta un clima frío semihúmedo ( $\mathrm{FsH})$, según la clasificación Caldas-Lang, que se basa en los valores de temperatura con respecto a su variación altitudinal [es decir, pisos térmi$\cos$ ] y no latitudinal, y la efectividad de la precipitación que muestra la humedad (Gutiérrez-Rey, 1989); registra una temperatura promedio mensual de $13,7 \pm 0,2{ }^{\circ} \mathrm{C}$ y una precipitación media anual de 853 $\mathrm{mm}$, para la estación climatológica de Gja. Providencia (Código 21205980 operada por el IDEAM), para el período 1987-2012 (26 años).

La distribución de la precipitación media mensual en la zona es de carácter bimodal, influenciada por la ZICT, que determina las condiciones atmosféricas y climáticas de la zona, provocando dos periodos de baja precipitación: entre junio y septiembre, y entre diciembre y marzo, y dos periodos lluviosos: el primero, entre abril y mayo, cuando la ZICT se desplaza hacia el norte, lo cual va acompañado de un pequeño descenso en las temperaturas máximas y un aumento de las mínimas (Eslava, 1993), y el segundo entre octubre y noviem- 
bre, cuando la ZITC regresa a su posición norte más extrema; este segundo periodo lluvioso es el más fuerte, acompañado por un descenso en las temperaturas medias, disminución de las temperaturas máximas y aumento de las mínimas (Eslava, 1993).

\subsection{Precipitación}

Se realizó un análisis exploratorio de datos, y se verificó la homogeneidad de los datos de todas las series pluviométricas utilizadas, a través de un análisis de doble masa $\left(\mathrm{r}^{2}>0,99\right)$. Las estaciones indican un promedio intermensual de 67,6 $\pm 3,4 \mathrm{~mm} / \mathrm{mes}$ (Figura 2).

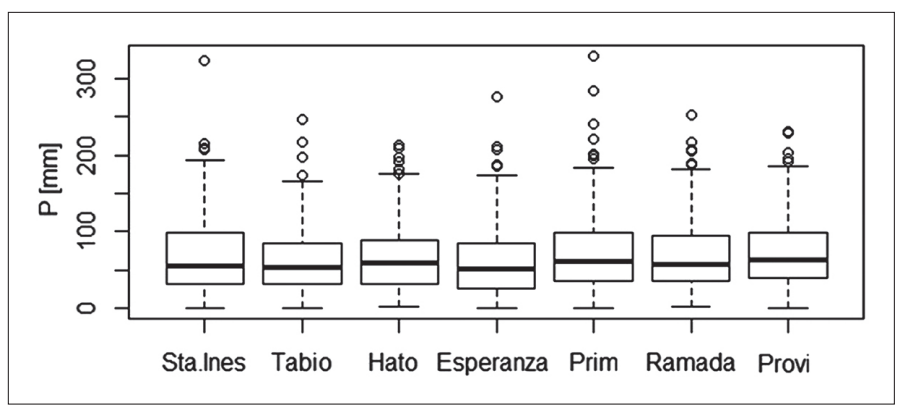

Hato: El Hato; Prim: La Primavera; Ramada: La Ramada; Provi: La Providencia

Figura 2. Boxplot Estaciones climatológicas seleccionadas en la zona de estudio.

Fuente: López (2015).

Posterior a la normalización y selección de las estaciones con mayor correlación $(\mathrm{r}>0,80)$ se realizó la estimación de datos faltantes en la serie pluviométrica de la estación Gja. Providencia, a través del método de regresión múltiple linear. El modelo estadístico escogido (Ecuación 8) para esta estimación responde sobre el $82 \%$ de la variabilidad de los datos analizados (adjusted $r^{2}=0,8217$ ).

$$
Y=0,91+0,28 X_{1}+0,35 X_{2}+0,29 X_{3}
$$

Donde:

$$
\begin{aligned}
Y & =\sqrt{P_{x \text { Providencia }}} \\
X_{1} & =\sqrt{P_{x \text { Sta.Ines }}} \\
X_{2} & =\sqrt{P_{x \text { Ramada }}} \\
X_{3} & =\sqrt{P_{x \text { Primavera }}}
\end{aligned}
$$

Para las demás estaciones analizadas se utilizó el método del cociente normal, es decir, con las estaciones que no superaron un coeficiente de correlación de $\pm 0,8$ y presentan una variación mayor al $10 \%$ entorno a los valores de precipitación 
media mensual. En la aplicación a la estación con el dato faltante de este método se tomaron las esta- (Figura 3), y fueron utilizadas con ciones pluviométricas que presen- el fin de generar los pesos estadístitaban mayor correlación respecto cos aplicados en el método.

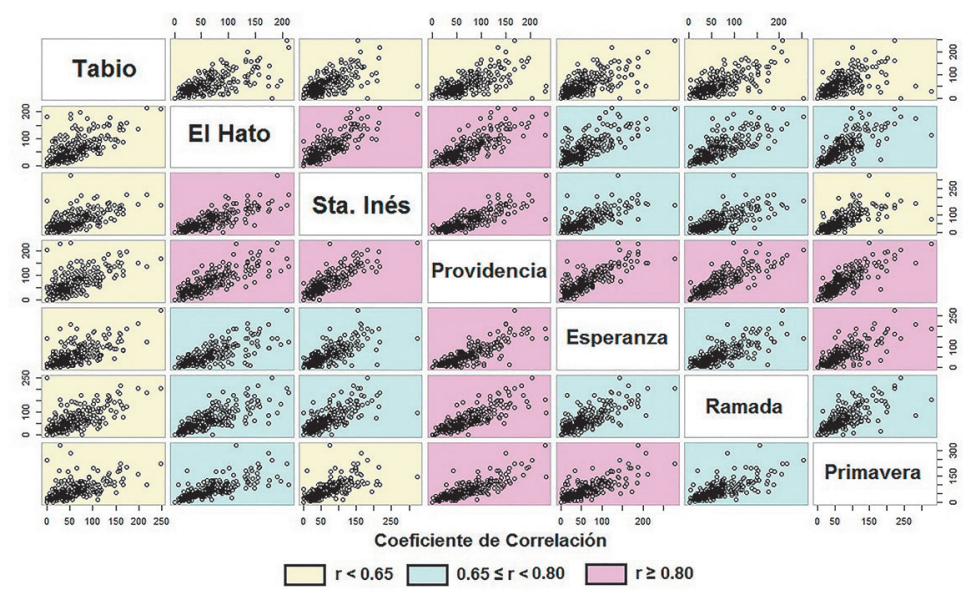

Hato. El: El Hato; Providencia: Gja. Providencia; Ramada: La Ramada; Primavera: La Primavera.

Figura 3. Variables ordenadas y coloreadas según correlación.

Fuente: López (2015).

Empleando series de precipitación minaron las precipitaciones mulcompletas de la misma longitud tianuales de todas las estaciones (1987-2012; 26 años), se deter- (Tabla 3).

Tabla 3. Estaciones climatológicas seleccionadas en la zona de estudio

\begin{tabular}{cccc}
\hline \multicolumn{3}{c}{ Datos de la estación } & $\overline{\mathbf{P}} \pm$ SD [mm/año] \\
\cline { 1 - 2 } Nombre & Tipo & Elev. (m.s.n.m.) & $\mathbf{( 1 9 8 7 - 2 0 1 2 )}$ \\
\hline Gja. Providencia & PM & 2556 & $749,7 \pm 156,8$ \\
Tabio & CP & 2620 & $705,7 \pm 263,2$ \\
Santa Inés & PM & 2551 & $790,1 \pm 206,5$ \\
El Hato & PM & 2575 & $852,9 \pm 177,4$ \\
\hline Esperanza & PM & 2556 & $709,3 \pm 247,2$
\end{tabular}




\begin{tabular}{cccc}
\hline \multicolumn{3}{c}{ Datos de la estación } & $\overline{\mathbf{P}} \pm$ SD [mm/año] \\
\cline { 1 - 3 } Nombre & Tipo & Elev. (m.s.n.m.) & $(\mathbf{1 9 8 7 - 2 0 1 2 )}$ \\
\hline La Primavera & CP & 2590 & $831,7 \pm 217,9$ \\
La Ramada & CP & 2545 & $787,5 \pm 201,3$ \\
\hline
\end{tabular}

Elev.: Elevación; CP: Estación Climatológica Principal; PM: Estación Pluviométrica; P: Precipitación; mm/año: milímetros por año; SD: Desviación Estándar

Fuente: Elaboración propia.

\subsection{Variabilidad interanual}

La precipitación media anual para el periodo 1987-2012 (26 años) en las estaciones que se encuentran dentro de la cuenca estudiada se detalla en la Tabla 4; en esta se encuentra que el valor mínimo medio es de 705,7 mm, correspondiente a la estación Tabio, ubicada en el extremo NE de la cuenca, y que el valor máximo medio es de 852,9 mm, para la estación El Hato, ubicada en la cuenca media de la región de estudio, mientras que el promedio regional es de $775,3 \mathrm{~mm}$, considerando todas las estaciones pluviométricas del área de estudio.
Para cada estación hidrometeorológica se calcularon diversos parámetros estadísticos que amplían la información sobre las características de las precipitaciones en el área de estudio. Las medidas de tendencia central y de dispersión permiten un mayor conocimiento de la variabilidad de los datos estadísticos (Puebla Gutiérrez, Rodríguez Rodríguez Eslava, 1993 Eslava, 1993 y Santos Preciado, 1995). En la Tabla 4 se presentan, para cada estación, los valores mínimos, medios y máximos, la desviación estándar y el coeficiente de variación anual.

Tabla 4. Medidas de tendencia central y de dispersión para las estaciones pluviométricas ubicadas en la zona de estudio

\begin{tabular}{ccccccc}
\hline Estación & $\begin{array}{c}\text { Mínima } \\
{[\mathbf{m m}]}\end{array}$ & $\begin{array}{c}\text { Media } \\
{[\mathbf{m m}]}\end{array}$ & $\begin{array}{c}\text { Máxima } \\
{[\mathbf{m m}]}\end{array}$ & $\begin{array}{c}\text { Mediana } \\
{[\mathbf{m m}]}\end{array}$ & $\begin{array}{c}\text { Desviación } \\
\text { estándar }[\mathbf{m m}]\end{array}$ & $\begin{array}{c}\text { Coeficiente de } \\
\text { variación [\%] }\end{array}$ \\
\hline $\begin{array}{c}\text { El Hato } \\
\text { Gja. }\end{array}$ & 537.3 & 852.9 & 1304.5 & 845.1 & 177.4 & 20.8 \\
$\begin{array}{c}\text { Providencia } \\
\text { Esperanza }\end{array}$ & 2246.1 & 749.7 & 1052.6 & 749.5 & 156.8 & 20.9 \\
\hline
\end{tabular}




\begin{tabular}{ccccccc}
\hline Estación & $\begin{array}{c}\text { Mínima } \\
{[\mathbf{m m}]}\end{array}$ & $\begin{array}{c}\text { Media } \\
{[\mathbf{m m}]}\end{array}$ & $\begin{array}{c}\text { Máxima } \\
{[\mathbf{m m}]}\end{array}$ & $\begin{array}{c}\text { Mediana } \\
{[\mathbf{m m}]}\end{array}$ & $\begin{array}{c}\text { Desviación } \\
\text { estándar [mm] }\end{array}$ & $\begin{array}{c}\text { Coeficiente de } \\
\text { variación [\%] }\end{array}$ \\
\hline Tabio & 254.1 & 705.7 & 1146.9 & 725.7 & 263.2 & 37.3 \\
Sta. Inés & 400.8 & 790.1 & 1314.0 & 767.6 & 206.5 & 26.1 \\
La Ramada & 456.1 & 787.5 & 1369.9 & 756.3 & 201.3 & 25.6 \\
$\begin{array}{c}\text { La } \\
\text { Primavera }\end{array}$ & 508.7 & 831.7 & 1477.5 & 821.9 & 217.9 & 26.2 \\
\hline
\end{tabular}

Fuente: Elaboración propia.

Los valores medios anuales mínimos y máximos de precipitación muestran los umbrales en los que varían los valores de una serie de años. Los valores mínimos anuales en la región de estudio se encuentran en las estaciones Esperanza (227,7 mm) y Tabio $(254,1 \mathrm{~mm}), \mathrm{y}$ los máximos en las estaciones Sta. Inés (1314,0 mm), E1 Hato (1.304,5 $\mathrm{mm})$ y Esperanza $(1.301,8 \mathrm{~mm})$ : las estaciones La Ramada y La primavera se encuentran fuera de la región de estudio, pero permiten conocer la variabilidad espacial de la precipitación en la zona. A partir de los valores observados anteriormente se puede afirmar que existe una importante variabilidad entre los montos mínimos y máximos, y su diferencia permite establecer que existe una variabilidad espacial de la precipitación en el área objeto de estudio.

Al graficar las series de precipitación anual para las estaciones de la cuenca (Figura 4 A, B y C), y compararlas con su media móvil a $5 \mathrm{y}$ 7 años, así como los años de niño y niña, cuyos valores medios fueron obtenidos del Golden Gate Weather Services (2015), se determinó que, en general, la precipitación anual en la cuenca ha aumentado progresivamente en el tiempo: entre los años 1987 y 2003 los valores oscilaron en el rango de $655,4 \pm 173,6$ $\mathrm{mm}$ (Est. Esperanza, precipitación media anual: 709,3 $\pm 247,2 \mathrm{~mm}$ ) y $\operatorname{los} 779,9 \pm 138,8 \mathrm{~mm}$ (Est. El Hato, precipitación media anual: $852,9 \pm$ $177,4 \mathrm{~mm}$ ), siendo valores inferiores a la media anual para 26 años; no obstante, en años subsiguientes (2004 a 2012) los valores de la media móvil para 5 y 7 años presentan tendencias positivas, superando los valores de media anual para los 26 años estudiados, encontrando valores medios anuales mínimos de 728,3 $\pm 351,8 \mathrm{~mm}$ (Est. Tabio) y máximos de 990,8 $\pm 164,7 \mathrm{~mm}$ (Est. El Hato). 
Por otra parte, en términos del comportamiento de la precipitación anual en la cuenca y el efecto del fenómeno del Niño-oscilación del Sur (ENSO, por sus siglas en inglés)-, en general, en el área de estudio (Tabla 5), en años de El Niño se produce una disminución de la precipitación anual, que no representa una sequía total, sino una disminución en el volumen de precipitación, más evidente en los periodos de baja precipitación de la región donde se presenta con mayor magnitud, y en años de La Niña se produce el fenómeno contrario al Niño: mayores precipitaciones, que se evidencian con mayor magnitud en los periodos de mayor precipitación. Estos fenómenos de variabilidad climática han sido determinados a través del Índice Oceánico del Niño (ONI, por sus siglas en inglés), que es una medida que sirve para identificar los eventos del Niño (cálido, ONI $>0$ ) y la Niña (frío, $\mathrm{ONI}<0$ ) en el trópico (Golden Gate Weather Services, 2015), donde el umbral se descompone como eventos: débiles (0.5$0.9)$, moderados $(1,0-1,4)$, fuertes $(1,5-1,9)$ y muy fuertes $(\geq 2,0)$.

Tabla 5. Años con fenómenos ENSO y precipitación anual en la región de estudio

\begin{tabular}{|c|c|c|c|c|c|c|c|c|c|c|c|}
\hline \multicolumn{6}{|c|}{ El Niño } & \multicolumn{6}{|c|}{ La Niña } \\
\hline Débil & $\mathrm{P}[\mathrm{mm}]$ & Mod. & $P[\mathrm{~mm}]$ & $\begin{array}{l}\text { Muy } \\
\text { Fuert } \\
\text { e }\end{array}$ & $\mathrm{P}[\mathrm{mm}]$ & Débil & $P[\mathrm{~mm}]$ & Mod. & $\mathrm{P}[\mathrm{mm}]$ & $\begin{array}{c}\text { Fuert } \\
\mathrm{e}\end{array}$ & $\mathrm{P}[\mathrm{mm}]$ \\
\hline 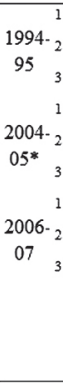 & $\begin{array}{c}859.0-739.6 \\
823.8-806.5 \\
7775.5-661.5 \\
356.9-254.1 \\
1010.9-906.4 \\
839.7-744 \\
406.8-986.6 \\
4023.2-877.0 \\
812.7-919.9\end{array}$ & $\begin{array}{r}1 \\
1987 \cdot{ }_{2} \\
88^{*} \\
3 \\
1 \\
1991-{ }_{2} \\
92 \\
3 \\
1 \\
2002-{ }_{2} \\
03 \\
3 \\
2009 . \\
10\end{array}$ & $\begin{array}{lll}1 & 534.8-1054.9 \\
2 & 729.3-959.2 \\
3 & 643.8-1052.6 \\
& 678.7-534.8 \\
1 & 678.7 \\
2 & 703.6-566.3 \\
3 & 726.6-480.3 \\
& 1 & 470.5-389.4 \\
2 & 678.6-756.2 \\
& 3 \\
3 & 662.8-755 \\
& 490.8-1146.9 \\
& 739.4-1136.3 \\
& 476.1-967.7 \\
\end{array}$ & $\begin{array}{r}1 \\
1997-{ }_{2} \\
98 \quad 3\end{array}$ & \begin{tabular}{|l|}
$561.1-783.5$ \\
$537.3-864.6$ \\
$564.7-907.5$
\end{tabular} & 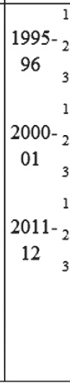 & $\begin{array}{lrl}1 & 739.6 & -987.7 \\
2 & 806.5 & -885.3 \\
3 & 661.5 & -691.3 \\
1 & 662.5 & -301.8 \\
2 & 782.1 & -661.5 \\
3 & 617.8 & -518.6 \\
1 & 1144.9 & -840.4 \\
2 & 1304.5 & -886.2 \\
3 & 916.6 & -831.8\end{array}$ & 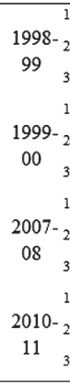 & $\begin{array}{lll}1 & 783.5 & -1022.7 \\
2 & 864.6 & -1093.7 \\
3 & 907.5-895.2 \\
1 & 1022.7-662.5 \\
2 & 1093.7-782.1 \\
3 & 895.2-617.8 \\
1 & 986.6-927.4 \\
2 & 877.0-1033.1 \\
3 & 919.9-951.6 \\
1 & 1146.9-1144.9 \\
{ }^{2} & 1136.3-1304.5 \\
3 & 967.7-916.6 \\
\end{array}$ & $\begin{array}{r}1 \\
1988 \cdot{ }_{2}^{1} \\
89 \\
3\end{array}$ & 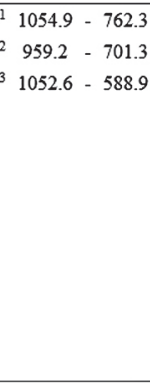 \\
\hline
\end{tabular}

* Esta lista se modificó en julio de 2015 para reflejar el uso de ERSSTv4 por el National Climatic Data Center en el cálculo de la Temperatura Superficial del Mar (TSM). En consecuencia, los eventos marcados con un asterisco se desplaza hacia abajo una categoría.

P [mm]: precipitación anual; Mod.: Moderado; 1.: Estación Tabio; 2.: Estación El Hato; 3.: Estación Gja. Providencia.

Fuente: Golden Gate Weather Services (2015) y Autor. 

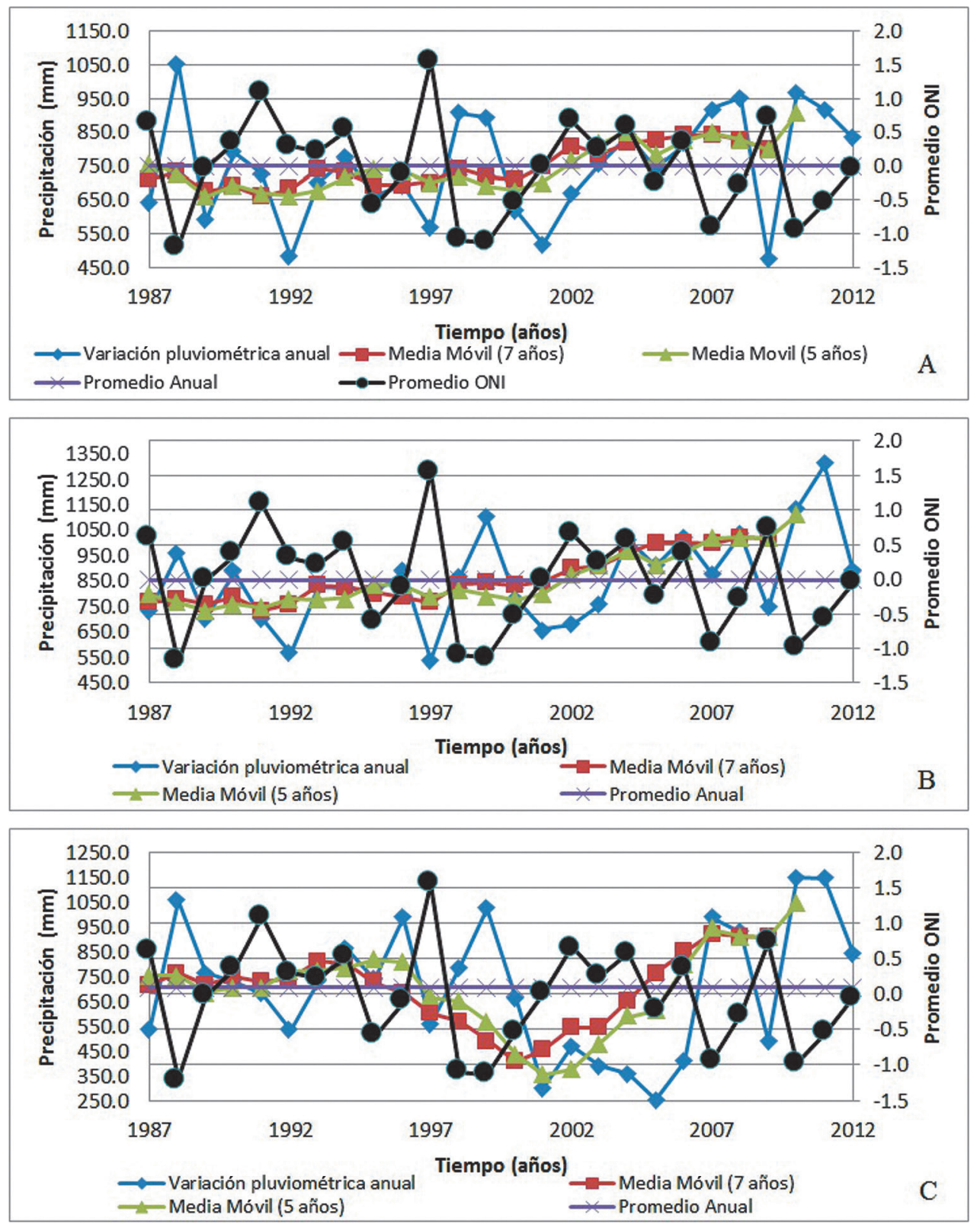

ONI: Índice oceánico del Niño

Figura 4. Precipitación anual y su relación con los fenómenos ONI: A) Estación Gja. Providencia; B) Estación El Hato; C) Estación Tabio

Fuente: Elaboración propia. 
5.3 Análisis estacional de la precipitación

Las estaciones Gja. Providencia (Cuenca Baja) y Tabio (Cuenca Alta) presentan un comportamiento de precipitación media mensual tipo bimodal (Figuras 5 y 6), con dos periodos lluviosos y dos de baja precipitación. Los períodos lluviosos ocurren entre abril y mayo $(101,9 \pm 15,6 \mathrm{~mm}$, estación Gja. Providencia; 82,9 $\pm 19,6$ mm, estación Tabio) y octubre y noviembre $(98,9 \pm 19,5 \mathrm{~mm}$, estación Gja. Providencia; 78,3 $\pm 17,5$ $\mathrm{mm}$, estación Tabio), siendo abril y octubre los meses más lluviosos: (102,2 $\pm 16,0 \mathrm{~mm}$, estación Gja. Providencia; $88,6 \pm 22,7 \mathrm{~mm}$, estación Tabio) y $(106,7 \pm 20,3 \mathrm{~mm}$, estación Gja. Providencia; 83,6 $6 \pm 16,2$ $\mathrm{mm}$, estación Tabio), respectivamente. Los períodos de baja precipitación ocurren de diciembre a febrero $(43,7 \pm 10,5 \mathrm{~mm}$, estación Gja. Providencia; 38,5 $\pm 12,4 \mathrm{~mm}$, estación Tabio) y de julio a septiembre $(55,8 \pm 12,1 \mathrm{~mm}$, estación Gja. Providencia; 54,3 $\pm 12,4 \mathrm{~mm}$, estación Tabio), siendo enero el mes más "seco" $(31,2 \pm 7,2 \mathrm{~mm}$, estación Gja. Providencia; 25,8 8 7,4 mm, estación Tabio).

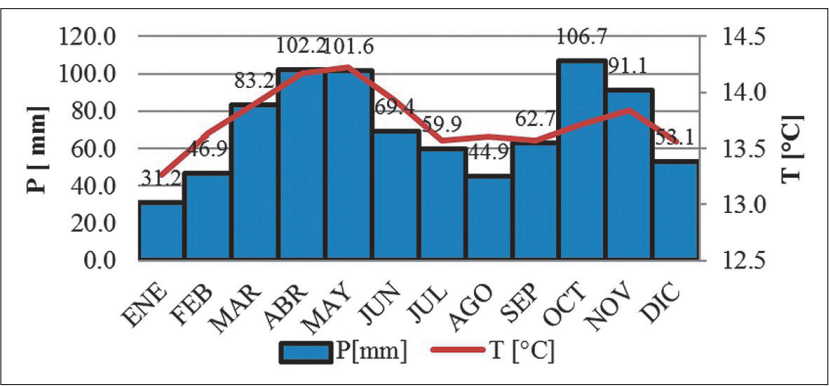

Figura 5. Climatograma de la estación Gja. Providencia Fuente: López (2015).

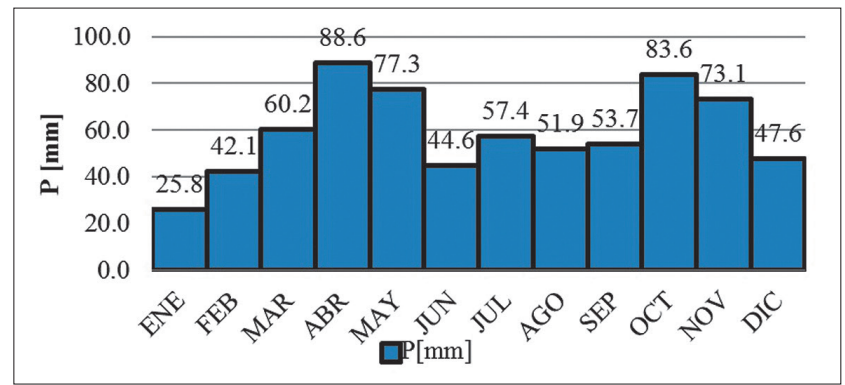

Figura 6. Hietograma de la estación Tabio

Fuente: López (2015). 
5.4 Elaboración de mapas de dis- las estaciones estudiadas y para la tribución espacial de la precipi- media intermensual, se ajustaron tación intermensual

Con base en la precipitación intermensual de los meses de julio y oclos criterios de convergencia para los datos analizados, obteniendo los siguientes parámetros de ajuste (Tabla 6).

tubre para los años 1987-2012 en

Tabla 6. Parámetros de Ajuste para la convergencia del modelo de Curvatura Mínima

\begin{tabular}{lccc}
\hline & Media interanual & Julio & Octubre \\
\hline Método grillado & Curvatura mínima & $\begin{array}{c}\text { Curvatura } \\
\text { mínima }\end{array}$ & $\begin{array}{c}\text { Curvatura } \\
\text { mínima }\end{array}$ \\
Residuo máximo & 0,013 & 0,015 & 0,026 \\
Iteración máxima & 100000 & 100000 & 100000 \\
Tensión interna & 0 & 0 & 0 \\
Tensión de borde & 0 & 0 & 0 \\
Factor de relajación & 1 & 1 & 1 \\
Relación de anisotropía & 0,6 & 1 & 0,7 \\
\hline
\end{tabular}

Fuente: Elaboración propia.

Con base en estos parámetros de calibración, se realizó la interpolación espacial de los datos a través del método de Curvatura Mínima, obteniendo los mapas de isohietas para la región en estudio (Figuras 7, 8 y 9$)$.

\subsection{Precipitación media inter- mensual (Normal: 26 años; 1987- 2012)}

Se observa una disminución generalizada de la precipitación en dirección NW-SE (Figura 7), produ- cida por los vientos dominantes de la región.

En la cuenca alta, la precipitación encuentra su mínimo (Est. Tabio: $58,8 \pm 10,5 \mathrm{~mm} / \mathrm{mes}) \mathrm{y}$, gradualmente, aumenta hasta la cuenca baja (Est. Gja. Providencia: 71,1 \pm $14,4 \mathrm{~mm} / \mathrm{mes}$ ). El comportamiento que se presenta en la zona de estudio es producido por el efecto que generan los ascensos orográficos que delimitan la región, los cuales, sumados a los vientos dominantes, ocasionan que la precipitación se 


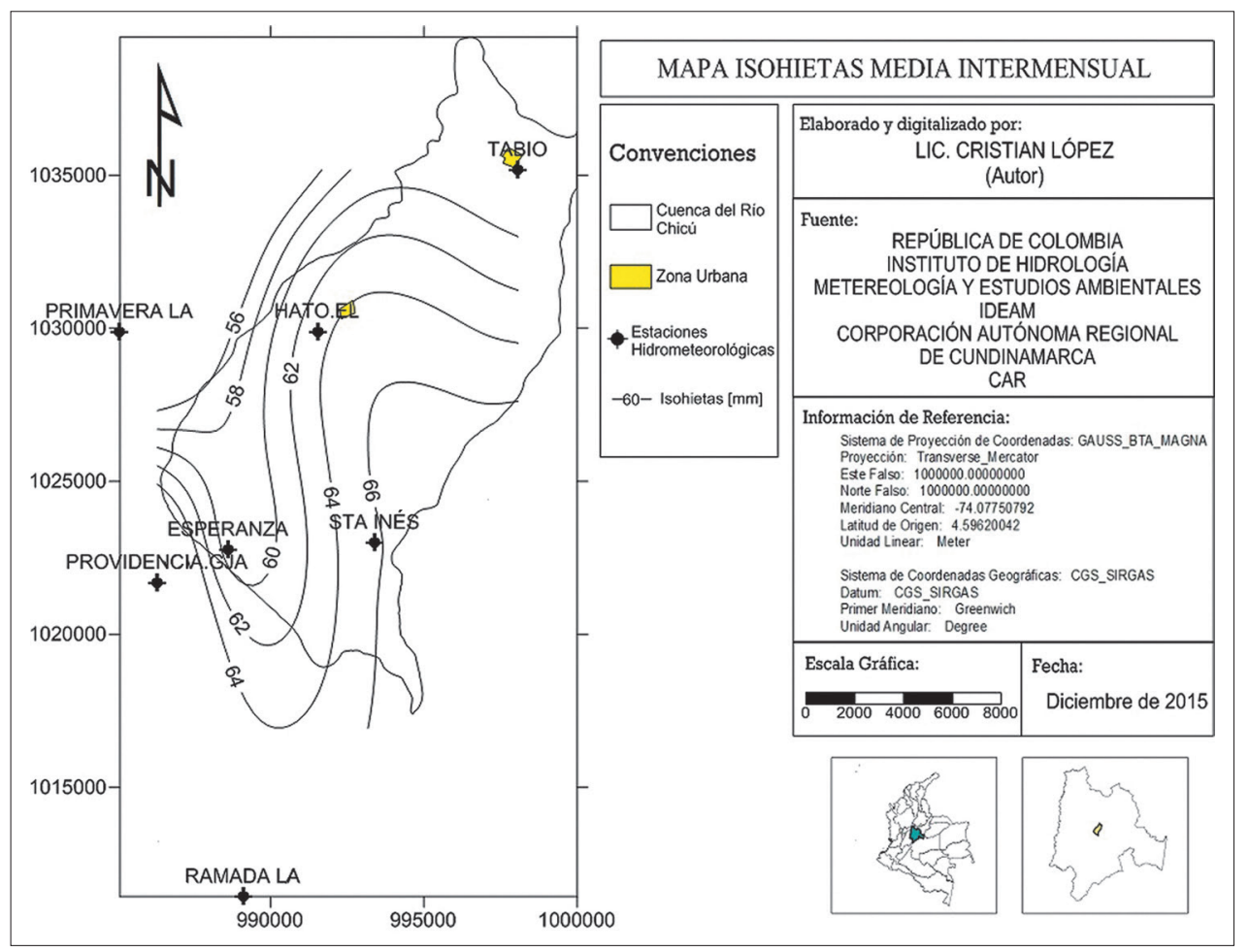

Figura 7. Mapa precipitación media intermensual

Fuente: Elaboración propia.

concentre en la cuenca media y en la serranía de Majuy, que limita el sector este de la cuenca, este efecto se evidencia en la estación Santa Inés $(65,8 \pm 14,1 \mathrm{~mm} / \mathrm{mes})$, próxima a la serranía de Majuy, la cual presenta valores de precipitación mayores, en comparación con las estaciones adyacentes.

A su vez, se evidencia una pluma de precipitación que no fue contenida por ningún límite orográfico en dirección NW-SE, que traspasa los límites de la cuenca del Chicú en el sector sur, dirigiéndose a la sabana bogotana para descargar en los municipios aledaños.

\section{Precipitación intermensual para} el mes de julio (Normal: 26 años; 1987-2012): En este mes se genera el segundo periodo de déficit hídrico del año, y se observa que sobre el área de estudio (Figura 8) la precipitación aumenta gradualmente en dirección de los vientos dominantes (NW-SE), en concordancia con el 
comportamiento evidenciado en la precipitación media intermensual. Los valores de mayor precipitación se concentran en los límites de la cuenca (Est. Tabio: $57,4 \pm 20,5 \mathrm{~mm} /$ mes; Est. Santa Inés: $53,7 \pm 22,7 \mathrm{~mm} /$ mes; Est. El Hato: 59,9 $20,1 \mathrm{~mm} /$ mes), donde se ubican los levantamientos orogénicos que delimitan la cuenca (serranía del Majuy) y valores de precipitación menores hacia el interior del valle intramontañoso, ubicados en la cuenca baja (Est. Esperanza: $45,5 \pm 22,0 \mathrm{~mm} / \mathrm{mes})$.



Figura 8. Mapa precipitación intermensual del mes de julio.

Fuente: Elaboración propia.

Precipitación intermensual para el mes de octubre (Normal: 26 años; 1987-2012): Este mes se encuentra dentro del segundo ciclo anual de excesos hídricos en la zona de estudio; en él (Figura
9), las precipitaciones presentan un aumento gradual del volumen en dirección (NW-SE), provocado por los vientos dominantes de la zona, que causan que los máximos de lluvia se generen en las zonas 
planas, ubicadas en la cuenca baja y media, por influencia de los ascensos orográficos que se presentan en la zona (Est. Esperanza: $93,7 \pm 29,3 \mathrm{~mm} / \mathrm{mes} ;$ Est. Santa
Inés: $108,9 \pm 37,3 \mathrm{~mm} / \mathrm{mes}$; Est. El Hato: $106,8 \pm 29,9 \mathrm{~mm} / \mathrm{mes})$, y los mínimos se presenten en la cuenca alta (Est. Tabio: $83,6 \pm 23,9 \mathrm{~mm} /$ mes).

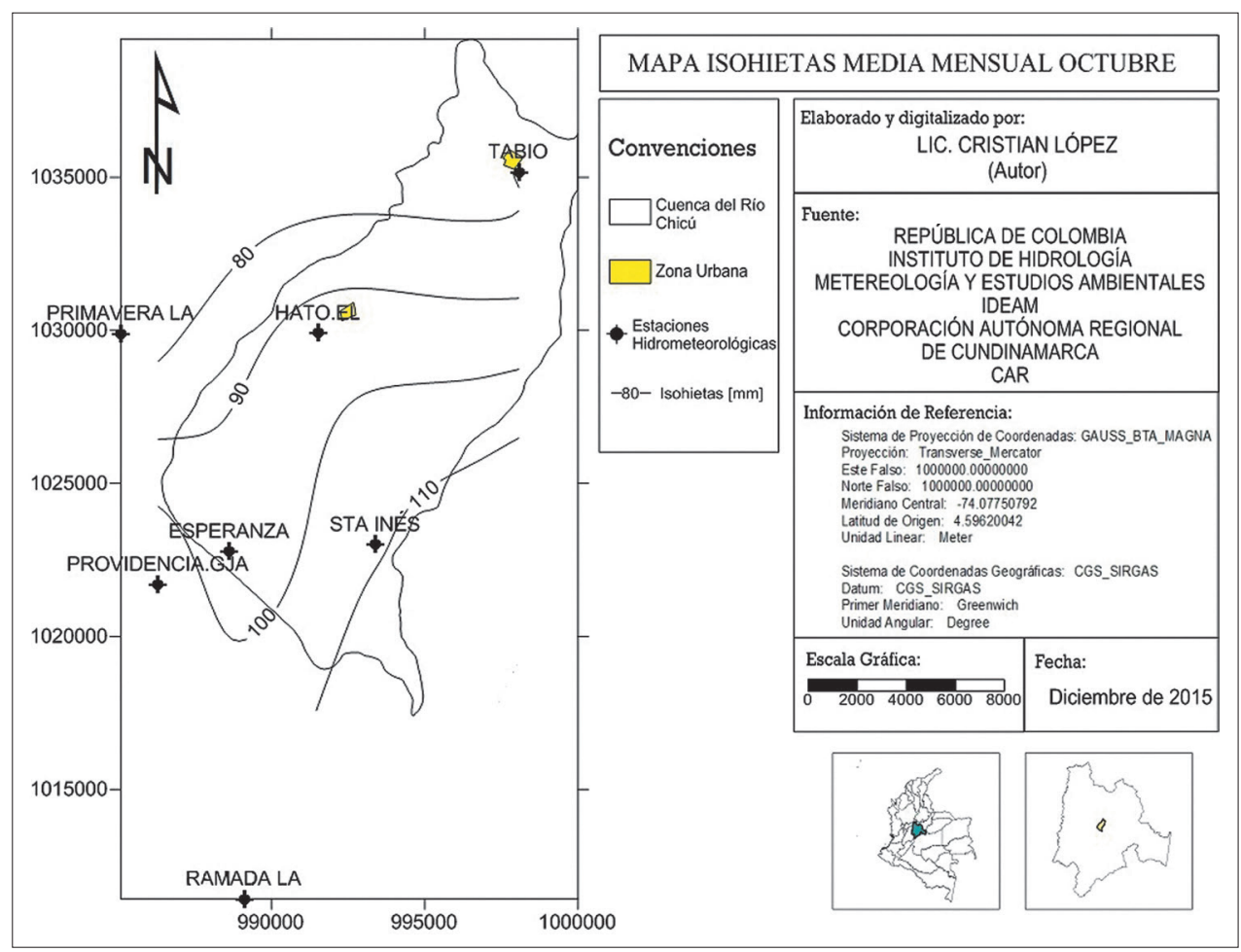

Figura 9. Mapa precipitación intermensual del mes de octubre.

Fuente: Elaboración propia.

\section{Conclusiones}

Dada la ubicación geográfica de la cuenca del río Chicú, y las características geomorfológicas $\mathrm{y}$ climáticas que presenta la zona de estudio, se puede concluir que esta presenta un clima frío semihúme- do $(\mathrm{FsH})$, según la clasificación Caldas-Lang, con una temperatura promedio mensual de $13,7 \pm 0,2{ }^{\circ} \mathrm{C}$ y una precipitación media anual de $853 \mathrm{~mm}$ para la estación climatológica de Gja. Providencia (Código 21205980, operada por el IDEAM), para el periodo de 1987- 
2012 (26 años). Por influencia de la ZICT, la distribución de la precipitación media mensual en la zona es de carácter bimodal, es decir, se generan dos periodos lluviosos: en los meses de abril-mayo y octubre. A través del análisis de las series de precipitación de las estaciones presentes en la zona (4 en el interior de la cuenca -densidad de red: $37,0 \mathrm{~km} /$ estación- y 3 complementarias, situadas alrededor de la cuenca) se logró describir la variabilidad espacial de la precipitación a escala municipal, y posterior a la realización del análisis exploratorio de datos, a la estimación de datos faltantes y a la verificación de la homogeneidad de datos $\left(\mathrm{r}^{2}>0,99\right)$, se obtuvo una marcada correlación $(r>0,80)$ en el comportamiento de la precipitación entre las estaciones El Hato-Sta. Inés-Providencia, Providencia-Esperanza-Ramada-Primavera y Esperanza-Rama$\mathrm{da}$; correlación que permitió realizar el análisis de la distribución de la precipitación en el área de estudio, para lo cual se emplearon series de precipitación completas de la misma longitud (1987-2012; 26 años) y se determinaron las precipitaciones multianuales de todas las estaciones.
En torno a la precipitación anual, se encuentran los valores mínimos anuales en las estaciones Esperanza $(227,7 \mathrm{~mm})$ y Tabio $(254,1 \mathrm{~mm})$, y los valores máximos anuales en las estaciones Sta. Inés (1314,0 $\mathrm{mm})$, El Hato $(1304,5 \mathrm{~mm})$ y Esperanza $(1301,8 \mathrm{~mm})$, encontrando una importante variabilidad entre los montos mínimos y máximos, cuya diferencia permite observar que existe una variabilidad espacial de la precipitación en el área objeto de estudio. Al comparar su comportamiento, usando la media móvil a 5 y 7 años, así como los años de El Niño y La Niña, se determinó que la precipitación anual en la cuenca ha aumentado progresivamente en el tiempo, con un ritmo acelerado entre los años 2004 y 2012, encontrando valores medios anuales mínimos de $728,3 \pm 351,8 \mathrm{~mm}$ (Est. Esperanza) y máximos de $990,8 \pm 164,7 \mathrm{~mm}$ (Est. El Hato), como rango medio para este lapso. Por otra parte, en términos del comportamiento de la precipitación anual en la cuenca con el fenómeno del Niño -oscilación del Sur-, en general, en el área de estudio en años de El Niño se produce una disminución de la precipitación anual, y en años de La Niña, un aumento. 
A nivel mensual, se obtuvieron en los períodos lluviosos valores de precipitación de 101,9 15,6 $\mathrm{mm}$ (estación Gja. Providencia) y $82,9 \pm 19,6 \mathrm{~mm}$ (estación Tabio), para los meses de abril-mayo, y 98,9 $\pm 19,5$ mm (estación Gja. Providencia) y $78,3 \pm 17,5 \mathrm{~mm}$ (estación Tabio), para los meses de octubre-noviembre, siendo abril, con 102,2 $\pm 16,0$ mm (estación Gja. Providencia) y $88,6 \pm 22,7 \mathrm{~mm}$ (estación Tabio), y octubre, con $106,7 \pm 20,3$ $\mathrm{mm}$ (estación Gja. Providencia) y $83,6 \pm 16,2 \mathrm{~mm}$ (estación Tabio), los meses más lluviosos. Los períodos de baja precipitación presentan valores de 43,7 $\pm 10,5 \mathrm{~mm}$ (estación Gja. Providencia) y $38,5 \pm 12,4 \mathrm{~mm}$ (estación Tabio) en los meses de

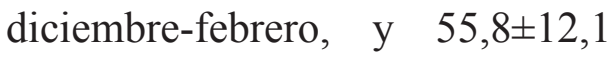
mm (estación Gja. Providencia) y $54,3 \pm 12,4 \mathrm{~mm}$ (estación Tabio) para los meses de julio-septiembre, siendo enero el mes más "seco" (31,2 $\pm 7,2$ mm, estación Gja. Providencia, y 25,8 $\pm 7,4 \mathrm{~mm}$, estación Tabio). Las estaciones escogidas representan la variabilidad de la precipitación en la cuenca baja y alta, respectivamente.

En torno a la distribución de precipitación en la cuenca, y teniendo en cuenta los datos promedio de la precipitación a lo largo de la serie
1987-2012 se observa un aumento generalizado de la precipitación en dirección NW-SE, correspondiente a la orientación de los vientos dominantes. A su vez, en la cuenca alta la precipitación encuentra su mínimo (Est. Tabio: 58,8 $\pm 10,5$ $\mathrm{mm} / \mathrm{mes}$ ), y gradualmente aumenta hasta la cuenca baja (Est. Gja. Providencia: 71,1 $\pm 14,4 \mathrm{~mm} / \mathrm{mes}$ ), comportamiento que se explica por el efecto que generan los ascensos orográficos que delimitan la región, provocando excesos hídricos en la serranía del Majuy, que limita la cuenca del río Chicú al este, lo cual se evidencia en la estación Santa Inés, que presenta regímenes de precipitación de mayor magnitud, comparados con los de las estaciones que la rodean, por efecto de la ubicación y características orográficas que la caracterizan.

Para el mes de julio, cuando se inicia el segundo periodo de déficit hídrico del año, se observa que la precipitación aumenta gradualmente en dirección de los vientos dominantes, encontrando en las estaciones presentes en la cuenca alta valores promedio de $57,0 \pm 3,1 \mathrm{~mm} /$ mes, donde se ubican los mayores levantamientos orogénicos que delimitan la cuenca, y valores de precipitación menores hacia el interior 
del valle intramontañoso, ubicados en la cuenca baja (Est. Esperanza: $45,5 \pm 22,0 \mathrm{~mm} / \mathrm{mes}$ ). Por otro lado, para el mes de octubre, que se encuentra dentro del segundo ciclo anual de excesos hídricos en la zona de estudio, se observa un aumento gradual del volumen precipitado en dirección (NW-SE) de los vientos dominantes de la zona que causan máximos de lluvia $(103,1 \pm 8,2 \mathrm{~mm} / \mathrm{mes})$ en las zonas planas, ubicadas en la cuenca baja y media, por influencia de los ascensos orográficos que se presentan en la cuenca; a su vez, se observan mínimos de lluvia (Est. Tabio: $83,6 \pm 23,9 \mathrm{~mm} / \mathrm{mes}$ ) en la cuenca alta, producto de la descarga sobre las zonas bajas de la cuenca.

Por último, cabe aclarar que los resultados expuestos son de especial interés para la planificación hidrológica a nivel municipal y de finca, ya que generan la necesidad de reflexionar la gestión del recurso agua en la cuenca, teniendo en cuenta el comportamiento estacional de la precipitación y los efectos de la variabilidad climática, que pueden afectar la productividad, la estabilidad de los suelos y el rendimiento de los cultivos, entre otros. No obstante, para entender la variabilidad en una escala nanoclimática (nivel finca) se recomendaría realizar estudios a esta escala. A su vez, con el fin de complementar el entendimiento de la variabilidad espacial de la región en estudio, se recomendaría la implementación de estaciones climatológicas principales en la cuenca alta, que permitieran conocer las variables climatológicas que se presentan en esta zona, con la finalidad de complementar la información para una adecuada planificación del recurso hídrico.

\section{Referencias}

Barbancho, A. C. (1998). Variabilidad espacial de la lluvia en una pequeña cuenca hidrográfica de la provincia de Cáceres. Norba. Revista de geografía, 10, 23-34.

Briggs, I. C. (1974). Machine Contouring Using Minimum Curvature. Geophysics, 39(1), 39-48.

Environmental Protection Agency. (2010). Climate change indicators in the United States. Recuperado de http://www.epa.gov/climatechange/indicators/pdfs/Climate Indicators_full. pdf 
Eslava, J. A. (1993). Climatología y Diversidad Climática de Colombia. Revista Académica Colombiana de Ciencias, 18(71), 507-538.

García, M. C., Piñeros, A., Bernal, F. A. y Ardilla, E. (2012). Variabilidad climática, cambio climático y el recurso hídrico en Colombia. Revista de Ingeniería - Universidad de los Andes, 36, 60-64.

Glandz, M. (2000). Currents of change El Niño's impacts on climate and society (Segunda ed.). United Kingdom: Cambridge University Press.

Golden Gate Weather Services (4 de diciembre de 2015). El Niño and La Niña Years and Intensities based on Oceanic Niño Index (ONI). Recuperado de http://ggweather.com/enso/ oni.htm

Golden Software, Inc. (2002). Surfe User's Guide: Contouring and 3D Surface Mapping for Scientists and Engineers. Colorado, USA: Golden Software, Inc.

Gutiérrez-Rey, H. J. (1989). Clasificaciones climáticas. Bogotá: Instituto Colombiano de Hidrología, Meteorología y Adecuación de Tierras.

Ideam, PNUD, MADS, DNP, Cancillería (2015). Nuevos escenarios de cambio climático para Colombia 2011-2100. Herramientas científicas para la toma de decisiones -enfoque nacional-departamental-: Tercera Comunicación Nacional de Cambio Climático. Bogotá, Colombia.

Lobo-Guerrero, A. (1992). Geología e Hidrogeología de Santafé de Bogotá y su Sabana. Ponencia presentada en la VII Jornada Geotécnicas de la Ingeniería de Colombia, 1-20.

López, C. (2015). Evaluación ambiental y química del recurso hídrico subterráneo de la cuenca del río Chicú, Bogotá, Colombia. La Plata, Argentina: Universidad Nacional de la Plata.

Mcelwain, L. y Sweeney, J. (2003). Climate change in Ireland. Recent trends in temperature and precipitation. Recuperado de http://www.ucd.ie/gsi/pdf/36-2/ climate.pdf. Departamento de Geografía, Universidad Nacional de Irlanda.

Medina, R. D. (2008). Estimación estadística de valores faltantes en series históricas de lluvia. Pereira, Colombia: Universidad Tecnológica de Pereira.

Mejía, F., Mesa, O., Poveda, G., Vélez, J., Hoyos, C., Mantilla, R. et al. (1999). Distribución espacial y ciclos anual y semianual de la precipitación en Colombia. Posgrado en Aprovechamiento de Recursos Hídricos, Universidad Nacional de Colombia.

Montoya, D. M. y Reyes, G. A. (2005). Geología de la Sabana de Bogotá. Bogotá: Instituto Colombiano de Geología y Minería.

Nogués-Paegle, J., y Mo, K. (1997). Alternating Wet and Dry Conditions over South America during Summer. Mon. Wea. Rev. 125, 279-291. 
Patrick, N., y Stephenson, D. (1990). Spatial variation of rainfall intensities for short duration storms. Hidrological Sciences -Journal-des Sciences Hydrologiques, 35, 667-680.

Poveda, G., Waylen, P. R., y Pulwarty, R. S. (2006). Annual and Inter-annual Variability of the Present Climate in Northern South America and Southern Mesoamerica. Palaeogeography, Palaeoclimatology, Palaeoecology, 234, 3-27.

Press, W., Flannery, B., Teukolsky, S., y Vetterling, W. (1988). Numerical Recipes in C. Cambridge, Reino Unido: Cambridge University Press.

Puebla Gutiérrez, J., Rodríguez Rodríguez, V., y Santos Preciado, J. (1995). Técnicas Cuantitativas (Estadística básica). Barcelona, España: Oikos-Tau.

Robles, E. y Sáenz, J. (1990). Hidrogeología de la Cuenca del río Chicú, Sabana de Bogotá. Bogotá: Instituto Nacional de Investigaciones Geológico-Mineras.

Robles, E., Martínez, C., Hincapié, G. y Álvarez, A. (1991). Estudio hidrogeológico cuantitativo de la Sabana de Bogotá, sector Tibitó-Salto de Tequendama y Oriente Bogotano. Bogotá: Instituto de Investigaciones en Geociencias, Minería y Química.

Rojas, E., Arce, B., Peña, A., Boshell, F. y Ayarza, M. (2010). Cuantificación e interpolación de tendencias locales de temperatura y precipitación en zonas alto andinas de Cundinamarca y Boyacá (Colombia). Revista Corpoica - Ciencia y Tecnología Agropecuaria, 11(2), 173182.

Smith, W., y Wessel, P. (1990). Gridding with Continuous Curvature Splines in Tension. Geophysics, 55(3), 293-305.

Waylen, P., Quesada, M., y Caviedes, C. (1996). Interannual variability of monthly precipitation in Costa Rica. Journal of Climate, 9, 2606-2613.

Yang, C. S., Kao, S. P., Lee, F. B., y Hung, P. S. (2004, Julio). Twelve different interpolation methods: A case study of Surfer 8.0. (Vol. 35, pp. 778-785). XXth ISPRS Congress. 35, 778785. Istambul: International society of photogrammetry and remote sensing.

Recepción: 26 de julio de 2015

Evaluación: 19 de febrero de 2016

Aceptación: 23 de febrero de 2016 